\title{
Two-stage Approach for Detecting Aberration of Influenza-like IIIness
}

\author{
Ta-Chien Chan*1, Yung-Chu Teng² and Jing-Shiang Hwang ${ }^{2}$ \\ ${ }^{1}$ Research Center for Humanities and Social Sciences, Academia Sinica, Taipei City, Taiwan; ${ }^{2}$ Institute of Statistical Science, \\ Academia Sinica, Taipei City, Taiwan
}

\section{Objective}

This study proposed a two-stage approach for early detection of aberrations of influenza-like illness (ILI) using the small-area based claim data of outpatient and emergency room visit.

\section{Introduction}

Pandemic 2009 H1N1 influenza and recent H7N9 influenza outbreaks made the public aware of the threat of influenza infection. In fact, annual influenza epidemic caused heavy disease burden and high economic loss around the world [1,2]. Although the virological surveillance provided the high sensitivity and specificity for testing results, the timeliness and the cost of the test were not feasible for extensive public health surveillance. In addition, traditional sentinel physician surveillance also encountered many challenges such as the representativeness and reporting bias. The seamless surveillance system without extra labor reporting would be the ideal approach. Taiwan had as high as $99 \%$ of health insurance coverage. The real-time monitoring of the ILI clinical visits in the communities could reflect the severity of influenza epidemics. In this study, we used an innovative two-stage approach for detecting aberrations during 2009 pandemic influenza in Taiwan.

\section{Methods}

The ILI was defined as $487.0,487.1$ and 487.8 in ICD-9 codes which was the most severe or specific definition [3]. The health insurance data from 2007 to 2009 was obtained from National Health Research Institute. At the first stage, the Poisson regression model and Zero-inflated Poisson regression model were used for outpatient and emergency room visits, respectively. The controlled factors included holidays, weekend, season and weather conditions. We used dynamic two-year temporal windows for modeling and predicting the next day's ILI visits and calculated the residuals, the differences between the observed and predicted visits, for further aberration signal detections. At the second stage, a modified Cusum method was used for detecting aberrations and alert signal would be labeled when consecutive three positive Cusum signals occurred.

\section{Results}

In the Figure 1, the sporadic signals in emergency room visits occurred in northern and central Taiwan during Chinese New Year holidays when most of the hospitals and clinics closed. The 2009 H1N1 pandemic flu epidemic in Taiwan began in August 2009. The consistent results were also found in the laboratory surveillance. After November 2009, there was no signal in the southern Taiwan where was the tropical climate area. The outpatient visits could detect signals two weeks earlier than emergency room visits in northern Taiwan.

\section{Conclusions}

The two-stage approach for detecting aberrations on outpatient visits and emergency room visits were both effective and complementary during pandemic 2009 H1N1 influenza in Taiwan. The small-area based surveillance could help public health intervention in the communities at the initial stage of epidemics.
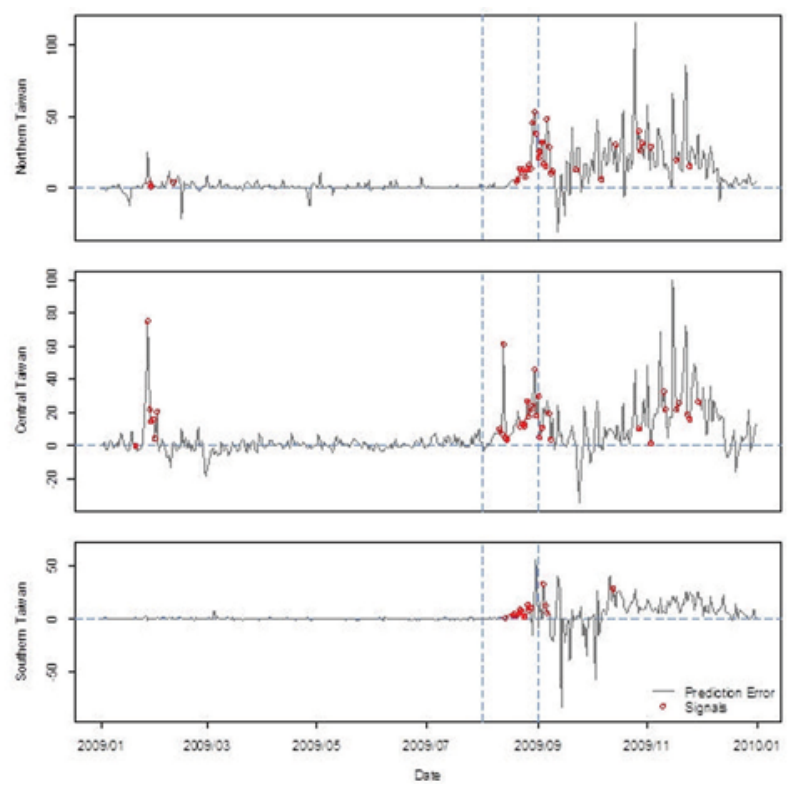

Figure 1 The Prediction Error of ILI Emergency Room Visits (2009)

\section{Keywords}

influenza surveillance; climate; Poisson model; zero-inflated Poisson; Cusum

\section{Acknowledgments}

This research was supported by a grant from the National Science Council, R.O.C. (NSC 101-2621-M-001-001).

\section{References}

References

1. Keech M, Beardsworth P: The impact of influenza on working days lost: a review of the literature. PharmacoEconomics 2008, 26(11):911924.

2. Paul Glezen W, Schmier JK, Kuehn CM, Ryan KJ, Oxford J: The burden of influenza B: a structured literature review. American journal of public health 2013, 103(3):e43-51.

3. Marsden-Haug N, Foster VB, Gould PL, Elbert E, Wang H, Pavlin JA: Code-based syndromic surveillance for influenzalike illness by International Classification of Diseases, Ninth Revision. Emerging infectious diseases 2007, 13(2):207-216.

\footnotetext{
*Ta-Chien Chan

E-mail: dachianpig@gmail.com
} 